\title{
Recent developments in totally asymmetric simple exclusion processes with local inhomogeneity
}

\author{
LIU MingZhe ${ }^{1 *}$, TUO XianGuo ${ }^{1,2^{*}}$, WANG RuiLi ${ }^{3} \&$ JIANG Rui ${ }^{4}$ \\ ${ }^{1}$ College of Nuclear Technology and Automation Engineering, Chengdu University of Technology, Chengdu 610059, China; \\ ${ }^{2}$ State Key Laboratory of Geohazard Prevention and Geoenvironment Protection, Chengdu University of Technology, Chengdu 610059, China; \\ ${ }^{3}$ School of Engineering and Advanced Technology, Massey University, Palmerston North 4442, New Zealand; \\ ${ }^{4}$ School of Engineering Science, University of Science and Technology of China, Hefei 230026, China
}

Received November 5, 2010; accepted December 21, 2010; published online April 13, 2011

\begin{abstract}
A totally asymmetric simple exclusion process (TASEP) has become an essential tool in modeling and analyzing non-equilibrium systems. A wide variety of TASEP models have been developed that are motivated by real-world traffic, biological transport and by the dynamics of the process itself. This paper provides an overview of recent developments in TASEP with inhomogeneity. Some important generalizations and extensions of inhomogeneous TASEP models are reviewed, and several popular mean-field techniques used to analyze the inhomogeneous TASEP models are summarized. A comparison between similar TASEP models under different updating procedures is given. Phase separations in such disordered systems have been identified. The present status of the inhomogeneous TASEP models and areas for future investigations are also described.
\end{abstract}

totally asymmetric simple exclusion process, inhomogeneity, mean-field theory, Monte Carlo simulations

Citation: Liu M Z, Tuo X G, Wang R L. et al. Recent developments in totally asymmetric simple exclusion processes with local inhomogeneity. Chinese Sci Bull,2011, 56: 1527-1531, doi: 10.1007/s11434-011-4449-4

\section{Introduction}

A totally asymmetric simple exclusion process (TASEP) has been acknowledged as a paradigmatic model for nonequilibrium systems. A TASEP is a one-dimensional lattice model in which particles hop to the nearest-neighbor sites in a preferred direction and interact through hard-core exclusion (i.e. each site can be occupied by no more than one particle at any given time). TASEP was introduced originally in 1968 as a theoretical model for describing ribosome motion along mRNA [1].

Recently, a wide variety of TASEP models have found natural applications in biology, physics, and chemistry $[2,3]$ such as gel electrophoresis [4], protein synthesis [5, 6], mRNA translation [7], motion of molecular motors along cytoskeletal filaments [8], and the depolymerization of

*Corresponding authors (email: liumz@cdut.edu.cn; txg@cdut.edu.cn) microtubules by special enzymes [9] as well as vehicular traffic $[10,11]$. Problems associated with these systems have motivated the development of many mathematical models.

Several review articles on TASEP models have been published from the perspective of applied biophysics [12, 13] as well as from a purely theoretical viewpoint $[14,15]$. We aim to provide an overview from a different perspective based on local inhomogeneities. In this paper, we give a brief review of some generalizations and extensions of the inhomogeneous TASEPs, and we summarize several popular mean-field techniques used to analyze inhomogeneous TASEP models. Phase separations previously observed in such disordered systems have been identified as well. Finally, we describe the present status of inhomogeneous TASEP models and areas for future investigations. TASEP variants have been successfully applied to modeling real-world complex systems in biology, physics and chemistry. However, because of the limited space in this paper, we do not review such applications in detail. There exists a large body of 
literature on TASEP-related applications. We refer the reader to review articles $[12,13]$ and references therein for biological transport, and review articles $[10,11]$ and references therein for vehicular traffic.

\section{TASEP with local inhomogeneities}

Local inhomogeneities are also referred to as site-wise inhomogeneities. They can be divided into single inhomogeneity and multiple inhomogeneities. An inhomogeneous site is normally characterized by a different hopping probability from that of homogeneous sites. In other words, if the hopping probability is denoted by $p, p=1$ represents normal sites, while $p \neq 1$ corresponds to inhomogeneous sites. In reality, local inhomogeneities may be involved in many biological transport processes [16] as well as in vehicular and pedestrian traffic [17]. For instance, the local inhomogeneity of immunoreactivity may lead to a high susceptibility to respiratory infection [16], while high-density (e.g. congested) vehicular traffic may be attributed to some local inhomogeneities, e.g. on-ramps, lane reductions or temporary road works [17]. Moreover, it has been proposed that the crowding of molecular motors may be a source of several human disorders including neurodegenerative and kidney diseases [18].

\subsection{TASEP with single inhomogeneity}

TASEP with single inhomogeneity in a one-dimensional lattice under open boundary conditions is illustrated in Figure 1. Such a system can be decomposed into two steps: (i) a single system with inhomogeneity is divided into two homogeneous subsystems, each of which is treated as a normal TASEP; (ii) the two subsystems are connected by this single inhomogeneous site. This approach is known as "Segmented Meanfield Approximation" (SMFA). The decomposition approach assumes that there are no correlations in the probability of finding two particles at any two sites, i.e.

$$
\left\langle\rho_{k} \rho_{k+1}\right\rangle=\left\langle\rho_{k}\right\rangle\left\langle\rho_{k+1}\right\rangle=\rho_{k} \rho_{k+1},
$$

where $\rho_{k}$ and $\rho_{k+1}$ represent statistical densities at sites $k$ and

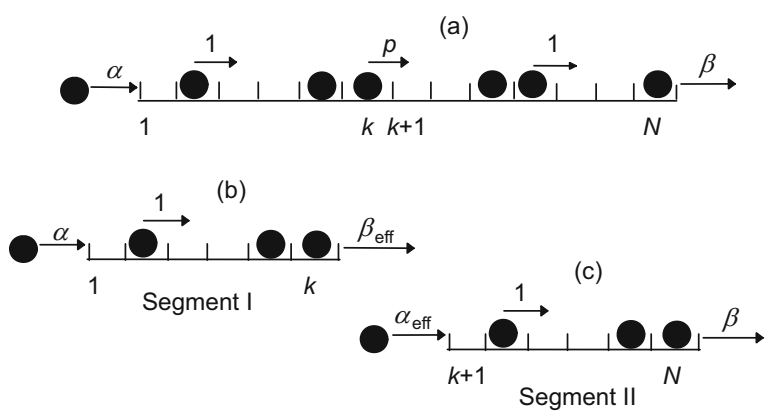

Figure 1 (a) Illustration of TASEP with a single inhomogeneity, which can be seen as two subsystems: (b) the left subsystem, and (c) the right subsystem. Arrows indicate that the movements are allowed. Numbers over arrows are hopping rates. $k+1$, respectively. $\langle\ldots\rangle$ is statistical average. This approach was first used to investigate TASEP with single inhomogeneity and random update [19]. In Figure $1, \beta_{e f f}, \alpha_{e f f}$ and the current $J_{k, k+1}$ between sites $k$ and $k+1$ can be written as

$$
\beta_{e f f}=p\left(1-\rho_{k+1}\right), \alpha_{e f f}=p \rho_{k}, J_{k, k+1}=p \rho_{k}\left(1-\rho_{k+1}\right) .
$$

$\beta_{e f f}$ and $\alpha_{e f f}$ are the exit rate and injection rate at the left and right subsystems, respectively. $\rho_{k}$ and $\rho_{k+1}$ are densities at sites $k$ and $k+1$. $p$ is the inhomogeneous hopping probability between sites $k$ and $k+1$. Theoretical analysis of the model in [19] indicates that the phase diagram is similar to that of the normal TASEP (i.e. the LD, HD and MC phases are similar between the models) [2] with shifts of phase boundaries. Table 1 lists the relationship between current and bulk density of different phases in the TASEP with single inhomogeneity and random update.

Similarly, TASEP with single inhomogeneity and parallel update has been studied in [20]. Parallel update exhibits strong interaction between particles, therefore, the model in [20] may be more suitable for car traffic, but less suitable for molecular motors or other biological transport. More importantly, eq. (2) cannot be used for theoretical analysis of parallel update. However, $J_{k, k+1}$ can be written as

$$
J_{k, k+1}=\frac{1}{2}\left(1-\sqrt{1-4 p \rho_{k}\left(1-\rho_{k+1}\right)}\right) .
$$

Applying the SMFA approach, TASEP with single inhomogeneity and parallel update can be solved analytically. Table 2 shows the corresponding density profiles and system currents in the LD, HD and MC phases. Compared to Table 1 , it is found that for the same $p$, current with parallel update is larger than that with random update. It is also shown that different updating procedures produce different dynamical properties even for the same system.

TASEP with single inhomogeneity and large particles has been investigated by Shaw et al. [21]. As expected, the phase diagrams are still qualitatively identical to the standard TASEP [2] with different phase boundaries. Large particles, also referred to as extended objects in the literature, can occupy several sites (i.e. particle size $\ell>1$ ). The counterparts of large particles in nature can be long-size vehicles, ribosomes, dimers, multiple-motor cooperative motion, large molecules or vesicles.

Foulaadvand et al. [22] studied a TASEP with variable hopping probability. The hopping probability is assumed to follow a binary or uniform distribution. These researchers found that the impact of disorder depends greatly upon the boundary conditions.

TASEP with local inhomogeneity coupled with Langmuir kinetics (often abbreviated to LK) in a one-dimensional lattice has been investigated in [23]. LK is widely used to describe absorption-desorption processes in surface chemistry. In biophysics, it is used to describe particles randomly attaching to and detaching from a lattice. In [23], a novel phase, termed the bottleneck phase (BP), is introduced to describe 
Table 1 Relation between current and bulk density of different phases in the TASEP with local inhomogeneity and random update [19]

\begin{tabular}{|c|c|c|c|}
\hline Phase & Conditions & Current $(J)$ & Bulk density $(\rho)$ \\
\hline LD & $\alpha<\beta, \alpha<p /(1+p)$ & $\alpha(1-\alpha)$ & $\rho_{L}=\rho_{R}=\alpha$ \\
\hline $\mathrm{MC}$ & $\alpha \geqslant p /(1+p), \beta \geqslant p /(1+p)$ & $p /(1+p)^{2}$ & $\rho_{L}=1 /(1+p), \rho_{R}=p /(1+p)$ \\
\hline
\end{tabular}

Table 2 Relation between current and bulk density of different phases in the TASEP with local inhomogeneity and parallel update [20]

\begin{tabular}{cccc}
\hline Phase & Conditions & Current $(J)$ & Bulk density $(\rho)$ \\
\hline LD & $\alpha<\beta, \alpha<p$ & $\alpha /(1+\alpha)$ & $\rho_{L}=\rho_{R}=\alpha /(1+\alpha)$ \\
HD & $\alpha>\beta, \beta<p$ & $\beta /(1+\beta)$ & $\rho_{L}=\rho_{R}=1 /(1+\beta)$ \\
MC & $\alpha \geqslant p, \beta \geqslant p$ & $p /(1+p)$ & $\rho_{L}=1 /(1+p), \rho_{R}=p /(1+p)$ \\
\hline
\end{tabular}

the current independently of boundary conditions. Several rich bottleneck-induced mixed phases (e.g. LD-BP, LD-MC$\mathrm{BP}, \mathrm{MC}-\mathrm{BP}-\mathrm{MC}$ ) are reported to result from the BP.

Apart from the study of the TASEP in a one-lane system, the effects of local inhomogeneity on two-lane TASEP coupled with LK are also considered [24]. A single inhomogeneity is assumed to be located in one of two lanes. It is found that the local inhomogeneity effect can be observed in both lanes because of particles changing to the other channel when the hopping probability of the inhomogeneous site is small. This effect can be reduced by increasing the hopping probability or channel-changing rate.

Theoretically, an inhomogeneous site can be found at any position of the lattice. In the above-mentioned investigations, the inhomogeneous site was assumed to be the middle of the lattice. It is expected that the phase diagram and density profiles are qualitatively the same for central and off-center inhomogeneous sites, provided the off-center site is distant from the boundaries.

\subsection{TASEP with multiple inhomogeneities}

Multiple inhomogeneities may include: (1) randomly distributed inhomogeneous sites; and (2) a group of continuous inhomogeneous sites. When several site-wise inhomogeneities exist, the system becomes more complex. Ref. [25] investigated dynamic properties of a TASEP with two different hopping rates $p_{a}$ and $p_{b}$ on a one-dimensional lattice. In this model, sites at rate $p_{b}$ are arranged with a period of $T$. The authors' theoretical analysis suggests that the dual-rate TASEP retains the three stationary phases (LD, HD and MC).

The effects of clustered slow codons (local inhomogeneities) in mRNA translation and protein synthesis are investigated using TASEP in [26]. Results from this study show that the clustered defects can affect ribosomal (particle) current. When the number of slow codons in a cluster exceeds 3 or 4, the ribosomal current is not significantly reduced. However, this approach does not allow segment lengths of more than 20 sites to be treated, on account of the increased numerical complexity for longer segments.

Dong et al. [27] investigated the effects of having inhomogeneities at different positions on a lattice. For instance, a slow site near the system boundaries induces a higher current than when the inhomogeneity is at the center of the lattice. Thus a concept of effective boundary rate is proposed. The corresponding effect, called "edge effect", implies a dependence of the current on the position of the inhomogeneity.

Differently from [27], [28] studied a case of a zoned inhomogeneity in the TASEP. The zoned inhomogeneity is a sequence of consecutive inhomogeneous sites with a reduced hopping probability $p(0<p<1)$. These consecutive inhomogeneities are assumed to be close to one of the boundaries. Theoretical and simulation results support each other and show that the current and the phase diagram depend not only on the length of the bottleneck, but also on its position. If two sequences of consecutive inhomogeneities appear in the system, it is found that inhomogeneities of smaller length and distant from the first one (near the boundary) have no influence on the maximal current (i.e. on transport capacity). This study provides alternative support for the edge effect.

Similarly, the effect of an extended inhomogeneity on a one-dimensional TASEP with parallel update was studied by Liu et al. [29]. Here, however, the extended inhomogeneity is assumed to be located in the middle of the lattice. The study considers two lattice geometries, $V$ and $W$. In Case $V$, the system consists of a left and right subsystem (segment I and segment II respectively). Segment I is a homogeneous lattice with hopping probability 1 , while segment II is also homogeneous, but with hopping probability $p$. Case $W$ is a natural extension of Case $V$, comprising three segments (I, II and III),corresponding to the left, middle and right subsystems, respectively.

The phase diagram of Case $W$ is shown in Figure 2(a). The (HD/MC/LD) phase means that the three segments (I, II and III) are in the HD, MC and LD phases, respectively. $\alpha^{*}$ and $\beta^{*}$ are a pair of critical points. Any change of $\alpha^{*}$ or $\beta^{*}$ will lead 

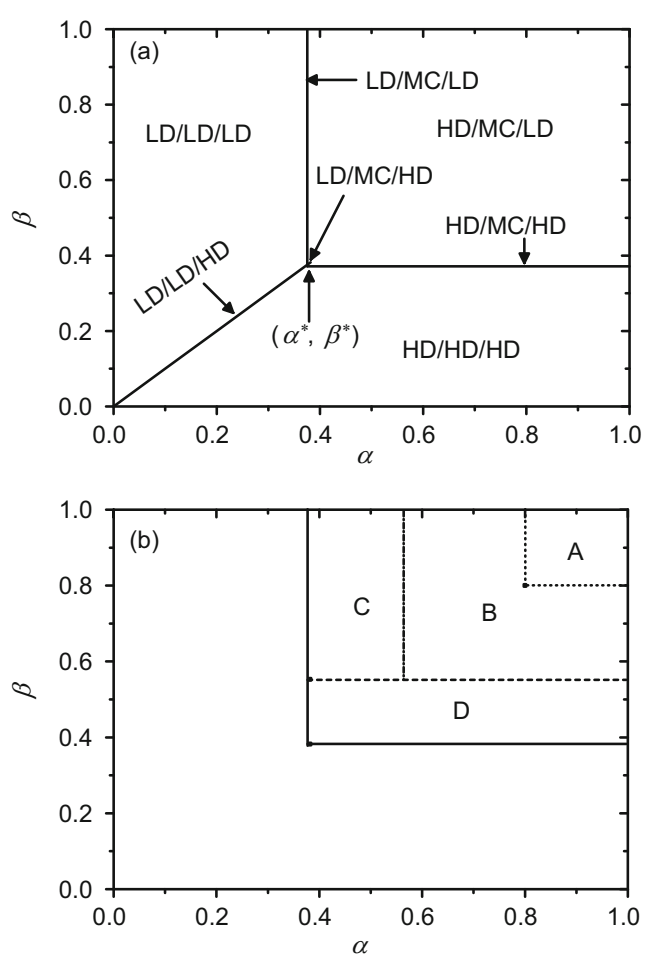

Figure 2 (a) Phase diagram of Case $W$ when $p=0.8$; (b) Comparisons of the $\mathrm{MC}$ regions with the following relationship: $\mathrm{A} \subseteq \mathrm{B}, \mathrm{B} \subseteq \mathrm{C}$, and $\mathrm{C} \subseteq \mathrm{D}$. See Table 3 for details of A, B, C, and D.

to a phase transition. The different $\mathrm{MC}$ regions are illustrated in Figure 2(b) and detailed in Table 3. From Figure 2(b), it can be seen that in case $W$ the $\mathrm{MC}$ region reaches the maximal area. However, the maximal currents are the same for the TASEP with zoned inhomogeneities (see Table 3). Another conclusion that can be drawn is that the maximal current of the TASEP with single inhomogeneity is larger than that of the TASEP with extended inhomogeneities because $p /(1+p)>(1-\sqrt{1-p}) / 2$ for $p<1$ (see Table 3$)$. Note that such models with random update have been studied by Xiao et al. [30]. Models with parallel update [29] and random update [30] have the same number of steady-state phases, but possess different phase boundaries. In addition, the maximal current in [29] is $(1-\sqrt{1-p}) / 2$, which is larger than that reported by [30], $p / 4$.

We remark that these recent investigations share two common features: (i) The presence of inhomogeneities leads to a decrease of the maximal current; however, the maximal- current regions expand, compared to the normal systems; and (ii) The occurrence of phase separations, reported in other disordered systems in a previous literature review [31], has also been observed in the above-mentioned disordered systems.

In practice, TASEP has been applied to modeling traffic flow in a single-lane highway with ramps [32]. For a single ramp (either on-ramp or off-ramp), the bottleneck phenomenon does not exist in this model. Traffic jams before the ramp and free flow after the ramp are not observed. For two consecutive ramps, the outcome changes. In particular, when an on-ramp is placed before an off-ramp, the model predicts that the bottleneck effect emerges and that the flow between the two ramps saturates. No real traffic experiments have been reported to either confirm or reject these predictions.

\section{Summary and conclusions}

The various aspects of TASEP with inhomogeneities reviewed in this paper cover a relatively small portion of the problems related to non-equilibrium processes. The phenomena of phase separations and the expansion of the maximalcurrent regions have been observed in these disordered systems. Theoretical methods (e.g. mean-field approximation) introduced here and in previous literature reviews could provide a basis for further developments.

From the perspective of mathematical modeling, many further investigations could be undertaken. On the other hand, the spatial correlations in mean-field treatments are normally neglected, resulting in deviations of theoretical results from computer simulations. More recently, the use of correlation functions to examine the spatially correlated variability, in particular for inhomogeneity cases, has been considered by Foulaadvand et al. [33]. Also recently, Sun et al. [34] investigated the effect of information feedback on traffic current in the presence of a single inhomogeneity. Such a model considers the next-step traffic and is resolved via computer simulations, the complexity of the model precluding analytical solutions. Model complexity in realistic scenarios poses a challenge to theoretical approaches. However, pursuing numerical approaches in future work is expected to reveal a rich variety of new phenomena.

In conclusion, we have highlighted the use of TASEP variants in the modeling and analysis of non-equilibrium systems,

Table 3 Details of Figure 2(b)

\begin{tabular}{ccc}
\hline Region & Number and position of inhomogeneous sites & MC \\
\hline A & an inhomogeneous site far away from boundaries & $p /(1+p)$ \\
B & all sites are inhomogeneous & $(1-\sqrt{1-p}) / 2$ \\
C & case $V$ & $(1-\sqrt{1-p}) / 2$ \\
D & case $W$ & $(1-\sqrt{1-p}) / 2$ \\
\hline
\end{tabular}


though the models are still oversimplified and remain unrealistic because of the lack of empirical data. Nevertheless, the simple TASEP models have proved encouraging enough for researchers to try and tackle more sophisticated models that reveal previously little unexplored phenomena. Future studies along these lines will hopefully provide deeper insight into fundamental issues as well as enhanced understanding of real-life traffic flows.

The authors gratefully acknowledge the comments and suggestions of the anonymous reviewers, which helped in improving the clarity and the quality of the paper. This work was supported by the National Natural Science Foundation for Distinguished Young Scholar (41025015), the National Natural Science Foundation of China (40974065, 10872194 and 11072239) and the Key Project of Natural Science of the Department of Education of Sichuan Province (10ZA110).

1 MacDonald C T, Gibbs J H, Pipkin A C. Kinetics of biopolymerization on nucleic acid templates. Biopolymers, 1968, 6: 1-25

2 Derrida B, Domany E, Mukamel D. An exact solution of one dimensional asymmetric exclusion model with open boundaries. J Stat Phys, 1992, 69: 667-687

3 Schütz G M. Phase Transitions and Critical Phenomena. San Diego: Academic Press, 2001

4 Widom B, Viovy J L, Defontaines A D. Repton model of gel electrophoresis and diffusion. J Phys I, 1991, 1: 1759-1784

5 Schütz G M. The Heisenberg chain as a dynamical model for protein synthesis - Some theoretical and experimental results. Int J Mod Phys B, 1997, 11: 197-202

6 Shaw L B, Zia R K P, Lee K H. Totally asymmetric exclusion process with extended objects: A model for protein synthesis. Phys Rev E, 2003, 68: 021910

7 Chou T. Ribosome recycling, diffusion and mRNA loop formation in translational regulation. Biophys J, 2003, 85: 755-773

8 Klumpp S, Lipowsky R. Traffic of molecular motors through tube-like compartments. J Stat Phys, 2003, 113: 233-268

9 Klein G A, Kruse K, Cuniberti G, et al. Filament depolymerization by motor molecules. Phys Rev Lett, 2005, 94: 108102

10 Chowdhury D, Santen L, Schadschneider A. Statistical physics of vehicular traffic and some related systems. Phys Rep, 2000, 329: 199-329

11 Helbing D. Traffic and related self-driven many-particle systems. Rev Mod Phys, 2001, 73: 1067-1141

12 Chowdhury D, Schadschneider A, Nishinari K. Physics of transport and traffic phenomena in biology: From molecular motors and cells to organisms. Phys Life Rev, 2005, 2: 318-352

13 Lipowsky R, Chai Y, Klumpp S, et al. Molecular motor traffic: From biological nanomachines to macroscopic transport. Physica A, 2006, 372: $34-51$

14 Schütz G M. Critical phenomena and universal dynamics in one- dimensional driven diffusive systems with two species of particles. J Phys A, 2003, 36: R339-R379

15 Blythe R A, Evans M R. Nonequilibrium steady states of matrix-product form: A solver's guide. J Phys A, 2007, 30: R333-R441

16 Estrella B, Estrella R, Oviedo J, et al. Acute respiratory diseases and carboxyhemoglobin status in school children of Quito, Ecuador. Environ Health Perspect, 2005, 113: 607-611

17 Neubert L, Santen L, Schadschneider A, et al. Single-vehicle data of highway traffic: A statistical analysis. Phys Rev E, 1999, 60: 6480-6490

18 Aridor M, Hannan L A. Traffic jams II: An update of diseases of intracellular transport. Traffic, 2002, 3: 781-790

19 Kolomeisky A B. Asymmetric simple exclusion model with local inhomogeneity. J Phys A, 1998, 31: 1153-1164

20 Liu M, Wang R, Jiang R, et al. Defect-induced transitions in synchroneous asymmetric exclusion processes. Phys Lett A,2009, 373: 195-200

21 Shaw L B, Kolomeisky A B, Lee K H. Local inhomogeneity in asymmetric simple exclusion processes with extended objects. J Phys A, 2004, 37: 2105-2113

22 Foulaadvand M E, Chaaboki S, Saalehi M. Characteristics of the asymmetric simple exclusion process in the presence of quenched spatial disorder. Phys Rev E, 2007, 75: 011127

23 Pierobon P, Mobilia M, Kouyos R, et al. Bottleneck-induced transitions in a minimal model for intracellular transport. Phys Rev E, 2006, 74 031906

24 Wang R, Liu M, Jiang R. Local inhomogeneity in two-lane asymmetric simple exclusion processes coupled with Langmuir kinetics. Physica A 2008, 387: 457-466

25 Lakatos G, Chou T, Kolomeisky A B. Steady-state properties of a totally asymmetric exclusion process with periodic structure. Phys Rev E, 2005, 71: 011103

26 Chou T, Lakatos G. Clustered bottlenecks in mRNA translation and protein synthesis. Phys Rev Lett, 2004, 93: 198101

27 Dong J J, Schmittmann B, Zia R K P. Inhomogeneous exclusion processes with extended objects: The effect of defect locations. Phys Rev E, 2007, 76: 051113

28 Greulich P, Schadschneider A. Phase diagram and edge effects in the ASEP with bottlenecks. Physica A, 2008, 387: 1972-1986

29 Liu M, Wang R, Hu M B, et al. Synchronous asymmetric exclusion processes with an extended defect. Phys Lett A, 2010, 374: 1407-1413

30 Xiao S, Cai J J, Liu F. Zone inhomogeneity with the random asymmetric simple exclusion process in a one-lane system. Chin Phys B, 2009, 18 : 4613-4621

31 Krug J. Phase separation in disordered exclusion models. Braz J Phys, 2000, 30: 97-104

32 Huang D W. Analytical results of asymmetric exclusion processes with ramps. Phys Rev E, 2005, 72: 016102

33 Foulaadvand M E, Kolomeisky A B, Teymouri H. Investigation of asymmetric exclusion processes with disorder: Effect of correlations. Phys Rev E, 2008, 78: 061116

34 Sun X Y, Wang B H, Yang H X, et al. Effects of information feedback on an asymmetrical two-route scenario. Chinese Sci Bull, 2009, 54: 3211-3214

Open Access This article is distributed under the terms of the Creative Commons Attribution License which permits any use, distribution, and reproduction in any medium, provided the original author(s) and source are credited. 